\title{
Risk of Thrombosis in Sjögren Syndrome: The Open Question of Endothelial Function Immune-mediated Dysregulation
}

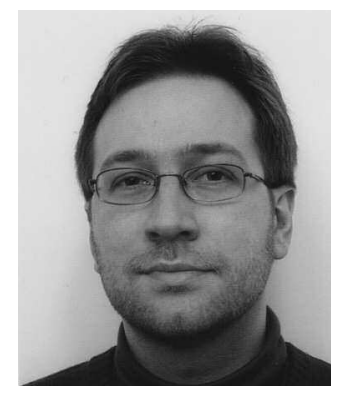

Deep vein thrombosis (DVT) and pulmonary embolism (PE) are the 2 common clinical expressions of the vascular phenomenon of venous thromboembolism (VTE). VTE is a common complication during and soon after hospitalization for acute medical illness or surgery ${ }^{1}$. In Europe, it is estimated to account for 60,000 deaths per year ${ }^{2}$, and because $\mathrm{PE}$ accounts for $5 \%-10 \%$ of deaths in hospitalized patients, VTE is the most common preventable cause of inpatient death ${ }^{1}$.

Historically, Virchow proposed 3 precipitants for venous thrombosis: venous stasis, increased coagulability of the blood, and damage to the vessel wall ${ }^{3}$.

Patients with systemic inflammatory diseases are at high risk for VTE because inflammation is a key determinant of endothelial dysfunction in both arteries and veins, by changing the expression of selectins and cellular adhesion molecules ${ }^{4}$ and leading to a state of hypercoagulability by influencing clotting factor levels ${ }^{5}$. Thus, systemic inflammation likely contributes to increasing the risk of VTE in addition to the other known factors, such as age, obesity, immobilization, and malignancy, and it is a possible trigger factor for venous thrombus formation.

Indeed, it is well known that an increased serum level of $\mathrm{C}$-reactive protein is an additional risk factor for cardiovascular (CV) events ${ }^{6}$. In fact, patients with a chronic inflammatory condition, such as those with rheumatoid arthritis (RA) or other chronic inflammatory arthritides, show a higher risk of $\mathrm{CV}$ events than the general population ${ }^{7}$.

Importantly, patients having an immune-mediated disease appear at risk of VTE, even independently from a detectable inflammatory state. In fact, in a record-linkage study on 2 UK datasets, the increased risk of VTE after hospital admission was reported not only for systemic autoimmune diseases usually characterized by laboratory signs of systemic inflammation such as RA, but also for systemic autoimmune diseases that do not usually show systemic inflammation, such as psoriasis, dermatomyositis, or polymyositis. The link was even seen for organ-specific autoimmune diseases such as autoimmune hemolytic anemia, type 1 diabetes mellitus, multiple sclerosis, myasthenia gravis, or autoimmune hepatitis $^{8}$. While the highest level of risk for VTE was recorded for systemic lupus erythematosus (SLE) and polyarteritis nodosa, consistently high levels of risk were also found for myositis, multiple sclerosis, and Sjögren syndrome $(\mathrm{SS})^{8}$.

A metaanalysis study on the risk of VTE in inflammatory rheumatic diseases concluded that overall, patients with RA, SLE, SS, myositis, systemic sclerosis, or antineutrophil cytoplasmic antibody-related vasculitis have a risk of VTE $3 \times$ higher than the general population. The increased risk was seen in studies of hospitalized and nonhospitalized patients. Some of the studies included in the metaanalysis also showed that the risk of VTE is highest in the first year of disease, with a significant dropoff later, leading to the hypothesis that effective medications could be involved in substantially decreasing the risk of VTE by controlling the systemic inflammation. In fact, in systemic vasculitides, thrombosis is a mirror of the endothelial hyperactivation because of an active state of disease, and the risk is lower when the disease undergoes an effective treatment ${ }^{9}$. However, the available methods to detect systemic inflammation are probably not sensitive enough because patients even in remission carry a higher risk of thrombosis ${ }^{10}$, or alternatively, the endothelial dysfunction may not be adequately targeted by the current therapies and a continuous low-grade procoagulant state persists despite a clinically effective treatment.

In the current issue of The Journal, Aviña-Zubieta, et a ${ }^{11}$ have published a general population-based study on the risk of DVT and PE in primary SS (pSS). This is a large general population-based study that specifically focused on the risk of DVT and PE in pSS. The study was designed as an incident (and not prevalent) cohort analysis of VTE among individuals with $\mathrm{pSS}$ as compared with individuals matched

See VTE in SS, page 1184

Personal non-commercial use only. The Journal of Rheumatology Copyright (C) 2017. All rights reserved. 
for age, sex, and entry time without pSS, selected from the general population using a province-wide database generated from the British Columbia, Canada, healthcare system. The International Classification of Diseases-9-Clinical Modification codes were used. The authors confirmed that pSS is associated with a statistically increased risk of VTE in a general population context, with HR of 4.07, 2.80, and 2.92 for PE, DVT, and VTE, respectively. Notably, the study also supports the notion that the risk is much higher during the first year after diagnosis $(8.29,4.72$, and 7.34 for PE, DVT, and VTE, respectively). The major limitations are the absence of verification of individual medical records, and the specific demographic and immunologic characterization of the pSS population under study. Nevertheless, the study is methodologically corrected, and it included a large sample size of 1175 incident pSS cases and 11,947 controls, adjustment for preexisting comorbidities and comedications, and 2 sensitivity analyses for the competing risk of death and unmeasured confounders in a general population context.

Now, the crucial question is how we can translate the clinical validity of these results into clinical utility. In this context, the magic words are "prevention" and "prophylaxis." However, there are limited data in the literature regarding these issues in the rheumatic diseases. The great majority of our knowledge at present is on SLE and antiphospholipid antibodies (aPL). Hydroxychloroquine may be beneficial in reducing the incidence of VTE in $\mathrm{SLE}^{12}$, as well as aspirin in aPL-positive patients with SLE, in particular during pregnancy ${ }^{13}$. In pSS, aPL can sometimes be detected, but their weight as CV risk factor has still to be well defined, and it could be different from that observed in SLE ${ }^{14,15}$.

Further, pSS is a syndrome and thus a heterogeneous disease $^{16}$. Not all the patients with pSS carry the same risk of lymphoma development ${ }^{17}$. Similarly, probably not all the patients with pSS are at the same risk of VTE as emerged from the abovementioned metaanalysis, in which pSS showed the widest CI for VTE incidence $(2.18 \%, 95 \%$ CI $0.79-3.57 \%$ ) if compared with the other autoimmune diseases ${ }^{9}$. This feature is critical for coming back from bench to bedside.

Indeed, the CV risk in pSS is higher for those patients with central nervous system involvement, those using immunosuppressive treatments, or showing leukopenia ${ }^{18}$. Thus, the systemic activity of the disease should be taken into account and different subsets of the disease may disclose different risk profiles. In addition, the positive effect of systemic treatments in reducing the $\mathrm{CV}$ risk in pSS still needs to be clarified.

Overall, differentiating the patient population based on the clinical manifestations, the global systemic disease activity, the autoantibody profile (including the possible presence of aPL), and the grade of the inflammatory infiltrate at the biopsy level would be very important. Some generalized risk factors must also be recognized, such as smoking status, level of physical activity, and body mass index. This additional information should be integrated in future research to improve clinical practice (i.e., new recommendations on thromboprophylaxis in immune-mediated diseases), and to reach the goal of personalized medicine (i.e., characterization of individuals who need thromboprophylaxis).

Even in the absence of such information, the clinician must be aware that immune-mediated diseases, including pSS, can determine a prothrombotic state, and that the risk is highest early after the diagnosis. Because both the morbidity and mortality of patients are strongly affected by VTE, these observations can help the clinician to improve the prevention or prophylaxis strategies for VTE, and under particular conditions that, even transiently but significantly, can further increase the risk of VTE events.

Finally, the increased risk of VTE in pSS, a disease in which the systemic inflammation is usually absent, underlines the role of additional factors other than the upregulation of classic proinflammatory pathways on the dysregulation of the endothelial function in pSS. It further suggests the need to analyze in greater depth the interaction between endothelial cells and lymphocyte subpopulations in pSS, as well as the possible secondary effects of targeting therapies on this interaction ${ }^{19,20}$.

LUCA QUARTUCCIO, MD, PhD, Rheumatology Clinic, University of Udine, DAME, Udine, Italy.

Address correspondence to Dr. L. Quartuccio, Clinic of Rheumatology, University of Udine, DAME, Piazzale S. Maria Misericordia 15, 33100 Udine, Italy. E-mail: luca.quartuccio@asuiud.sanita.fvg.it

\section{REFERENCES}

1. Cohen AT, Tapson VF, Bergmann JF, Goldhaber SZ, Kakkar AK, Deslandes B, et al; ENDORSE Investigators. Venous thromboembolism risk and prophylaxis in the acute hospital care setting (ENDORSE study): a multinational cross-sectional study. Lancet 2008;371:387-94.

2. Cohen AT, Agnelli G, Anderson FA, Arcelus JI, Bergqvist D, Brecht JG, et al; VTE Impact Assessment Group in Europe (VITAE). Venous thromboembolism (VTE) in Europe. The number of VTE events and associated morbidity and mortality. Thromb Haemost 2007;98:756-64.

3. Virchow R. [Collected treatises on scientific medicine]. [Book in German] Frankfurt: Verlag von Meidlinger Sohn; 1856.

4. Xu J, Lupu F, Esmon CT. Inflammation, innate immunity and blood coagulation. Hamostaseologie 2010;30:5-6, 8-9.

5. Levi M, van der Poll T, Buller HR. Bidirectional relation between inflammation and coagulation. Circulation 2004;109:2698-704.

6. Magapu P, Haskard D, Fisher M. A review of the peri-operative risk stratification assessment tools used for the prediction of cardiovascular complications in non-cardiac surgery. Perfusion 2016;31:358-65.

7. Castañeda S, Nurmohamed MT, González-Gay MA. Cardiovascular disease in inflammatory rheumatic diseases. Best Pract Res Clin Rheumatol 2016;30:851-69.

8. Ramagopalan SV, Wotton CJ, Handel AE, Yeates D, Goldacre MJ.

Personal non-commercial use only. The Journal of Rheumatology Copyright (C) 2017. All rights reserved. 
Risk of venous thromboembolism in people admitted to hospital with selected immune-mediated diseases: record-linkage study. BMC Med 2011;9:1.

9. Lee JJ, Pope JE. A meta-analysis of the risk of venous thromboembolism in inflammatory rheumatic diseases. Arthritis Res Ther 2014;16:435.

10. Hilhorst M, Winckers K, Wilde B, van Oerle R, ten Cate H, Tervaert JW. Patients with antineutrophil cytoplasmic antibodies associated vasculitis in remission are hypercoagulable. $\mathrm{J}$ Rheumatol 2013;40:2042-6.

11. Aviña-Zubieta JA, Jansz M, Sayre EC, Choi HK. The risk of deep venous thrombosis and pulmonary embolism in primary Sjögren syndrome: a general population-based study. J Rheumatol 2017;44:1184-9.

12. Petri M. Hydroxychloroquine use in the Baltimore Lupus Cohort: effects on lipids, glucose and thrombosis. Lupus 1996;5 Suppl 1:S16-22.

13. Arnaud L, Mathian A, Ruffatti A, Erkan D, Tektonidou M, Cervera $\mathrm{R}$, et al. Efficacy of aspirin for the primary prevention of thrombosis in patients with antiphospholipid antibodies: an international and collaborative meta-analysis. Autoimmun Rev 2014;13:281-91.

14. Pasoto SG, Chakkour HP, Natalino RR, Viana VS, Bueno C, Lianza $\mathrm{AC}$, et al. Lupus anticoagulant: a marker for stroke and venous thrombosis in primary Sjögren's syndrome. Clin Rheumatol 2012;31:1331-8.
15. Fauchais AL, Lambert M, Launay D, Michon-Pasturel U, Queyrel $\mathrm{V}$, Nguyen N, et al. Antiphospholipid antibodies in primary Sjögren's syndrome: prevalence and clinical significance in a series of 74 patients. Lupus 2004;13:245-8.

16. Ferro F, Marcucci E, Orlandi M, Baldini C, Bartoloni-Bocci E. One year in review 2017: primary Sjögren's syndrome. Clin Exp Rheumatol 2017;35:179-91.

17. Quartuccio L, Isola M, Baldini C, Priori R, Bartoloni Bocci E, Carubbi F, et al. Biomarkers of lymphoma in Sjogren's syndrome and evaluation of the lymphoma risk in prelymphomatous conditions: results of a multicenter study. J Autoimmun 2014; 51:75-80.

18. Bartoloni E, Baldini C, Schillaci G, Quartuccio L, Priori R, Carubbi F, et al. Cardiovascular disease risk burden in primary Sjögren's syndrome: results of a population-based multicentre cohort study. J Intern Med 2015;278:185-92.

19. Mantovani A, Bussolino F, Dejana E. Cytokine regulation of endothelial cell function. FASEB J 1992;6:2591-9.

20. Moreira DM, da Silva RL, Vieira JL, Fattah T, Lueneberg ME, Gottschall CA. Role of vascular inflammation in coronary artery disease: potential of anti-inflammatory drugs in the prevention of atherothrombosis. Inflammation and anti-inflammatory drugs in coronary artery disease. Am J Cardiovasc Drugs 2015;15:1-11.

J Rheumatol 2017;44:1106-8; doi:10.3899/jrheum.170462 